Editorial

\title{
Sport Injury Rehabilitation and Role of Sports Physiotherapist
}

\section{Editorial}

Once sports were limited to leisure activities but now a day it has become professional event for athlete and entertainment for audience. This increase demand of sports cause different types of sports injuries. Some of them are less affective but majority of them retard athletic performance. There are some predictors for delay performance. From the best knowledge it may be due to either premature return to sports after injury or inadequate rehabilitation due to lack of expert sports physiotherapist (SPT) in this sector. Thus, the view came into the mind of scholars that sports injuries are manageable but requires early detection and appropriate rehabilitation in order to return in sports. The aim of sports injury rehabilitation is to enable the athlete to return to sports with full function in the shortest possible time. An expert SPT can detect the athlete problems earlier at the time of injury during on field examination. In addition, continuation of sports is an important question for team management and SPT are capable of identifying problems, taking decisions immediate after the injury occurs with consultation of team manager and coach. In modern sports medicine team, all members of the team works on patient centered care. The aim of each professional is to help the injured athlete to return sports as early as possible. Despite of each professional's unique role SPT help to prepare and maintain injured athletes stability,muscle conditioning, soft tissue extensibility, proprioception, functional exercise, sports specific skills, correction of abnormal biomechanics, and cardiovascular fitness. Muscle conditioning always focused on muscle strength, muscle power, muscle endurance and motor re-education. Soft tissue extensibility can be improved by continuous passive motion, passive mobilization, passive exercise, active exercise, active assisted exercise etc. As soon as muscle strength and flexibility is achieved proprioceptive exercises are begun by partial and full weight bearing, jumping, hopping, skipping and running drills. Functional exercises are practiced by sprinting, agility drills, figure of eight running etc. Sports skills are
Volume I Issue 3 - 2017

\author{
Mohammad Habibur Rahman \\ Assistant Professor, Department of Physiotherapy, Bangladesh \\ Health Professions Institute (BHPI), CRP, Bangladesh \\ Correspondence: Mohammad Habibur Rahman, Assistant \\ Professor, Department of Physiotherapy, BHPI, CRP, Chapain, \\ Savar, Dhaka-1343, Bangladesh, Email sumonpt1983@gmail.com
}

Received: May 13, 2017| Published: June 09, 2017

focused with some activities that related to specific demand of sport. Any sign of abnormal biomechanics are corrected and re-trained within shortest period of time. However, cardiovascular fitness is related to training and adopting cardiovascular capacity improvement up to most potential state of pre-injury level. The SPT usually delivers physiotherapeutic intervention based on knowledge and clinical reasoning. Besides, an important aspect should consider by the Board of Sports Event in each country to ensure SPT at the age level groups of player's so that players can set up their mind that a SPT can develop their rehabilitation plan after injury or injury prevention plan. Thus, the potentiality of players can be maximized and role of SPT is to be understandable to Board of Sports Event in a sports rising country like Bangladesh.

\section{Acknowledgments}

None.

\section{Conflict of interest}

The author declares no conflict of interest. 